\title{
An Epidemiological Model for Semantics Dissemination *
}

\author{
C. Anagnostopoulos \\ Dept. Informatics \& \\ Telecommunications \\ University of Athens \\ 15784, Athens, Greece \\ bleu@di.uoa.gr
}

\author{
E. Zervas \\ Dept. Electronics \\ TEl of Athens \\ 12210, Athens, Greece \\ zervas@ee.teiath.gr
}

\author{
S. Hadjiefthymiades \\ Dept. Informatics \& \\ Telecommunications \\ University of Athens \\ 15784, Athens, Greece \\ shadj@di.uoa.gr
}

\begin{abstract}
Bio-inspired computing models have gained significant popularity in the engineering of distributed and autonomous systems. Epidemics assist in the direction of collaborative computing where groups of nodes can collectively share semantic content based on the current mobile context and user interests. Semantic information received by a node could infect the node and improve the existing knowledge according to pre-existing semantic structure. The capability of a node to reason with semantic information results in locally inferred information, which becomes a new potential epidemic. The application of epidemiology and context - awareness on semantically enriched information dissemination in ad-hoc mobile networks is analyzed and assessed using simulations.
\end{abstract}

\section{Keywords}

Ad hoc network information dissemination, Autonomous Systems, Context awaremess, Epidemics

\section{INTRODUCTION}

Persons that move to different locations can disseminate information (e.g., multimedia content) based on the mobile context (e.g., location and network connection). As persons group together (e.g., in conferences), they must be of similar interest (e.g., group of persons interested in the same presentation). Collaborative context-awareness is an understanding of the activities / conditions / environmental parameters of others that, consequently, provides a more enhanced context for an individual. Context-aware applications generate inferred knowledge, which is needed by the rest of the group. Such applications have to adopt information dissemination algorithms (e.g., epidemical spreading) and exploit the ways in which users' behavior coincides with their interests[5]. We propose a method for collaborative information dissemination to a group of context-aware applications in a pervasive computing environment using an epidemiological

\footnotetext{
*Mobimedia'07, Month 8, 2007, Nafpaktos, Aitoloakarna-
} nia, Greece; Copyright 2007 ICST 978-963-06-2670-5

Permission to make digital or hard copies of all or part of this work for personal or classroom use is granted without fee provided that copies are not made or distributed for profit or commercial advantage and that copies bear this notice and the full citation on the first page. To copy otherwise, to republish, to post on servers or to redistribute to lists, requires prior specific permission and/or a fee. MOBIMEDIA 2007, August 27-29, Nafpaktos, Greece

Copyright (C) 2007 ICST 978-963-06-2670-5

DOI 10.4108/ICST.MOBIMEDIA2007.1723 model. Information dissemination can cover the dissemination of multimedia content (e.g., text, image, voice and video) and semantic content (e.g., mpeg7 files).

Disseminated information could match an epidemic in the sense that, a mobile node carrying a piece of information content becomes infectious; otherwise it is susceptible. Infectious node means that it can disseminate such content to its neighboring nodes according to mobile context and their interest. When a user or application removes such content then the node becomes susceptible and can be infected later. In this paper, the terms epidemic and semantic information are used interchangeably. We move beyond the application of a simple epidemiological model. Semantic multimedia content (e.g., mpeg7 files) and user profiles (e.g., ontologies of user preferences) could be exploited in order for a user to obtain the multimedia content that adequately matches to his interest.

Consider a user with a specific interest in music, e.g., a fan of Modern Jazz music type. If the user node has been infected with a Jazz music mp3 file then he could replace it with a Modern Jazz music mp3 file. That is because, the latter music type matches better to his music interest than the former one; we assume a music ontology in which a song classified as a Modern Jazz concept is also classified as a Jazz concept, i.e., Modern Jazz $\sqsubseteq J a z z$, where $\sqsubseteq$ is the is-a relation. In this context, the user is reinfected with a stronger epidemic, in the sense that, the latter concept (describing multimedia metadata) represents a music interest closer to his interests. Hence, the Modern Jazz music file is more preferable. Similarly, consider that a node has been infected with a file including only text and image content (e.g., a static html file reporting the events during a soccer match). This node can be possibly reinfected with a multimedia content if (i) there is another neighboring node carrying the same information in video content and (ii) the user prefers viewing video than static content in his Personal Digital Assistant. Consequently, several semantic relations among multimedia metadata (semantic annotations), e.g., $\sqsubseteq$, have to be taken into consideration in information dissemination. Therefore, a node becomes uninterested once it has recently received a more preferable multimedia content than the incoming one. We introduce the abstraction of a stronger epidemic than the one, which has previously infected a given node. Stronger epidemics improve the existing knowledge of a node according to pre-existing semantic structure (i.e., a conceptual hierarchy is adopted). Moreover, our epidemiological 
abstraction of a context-aware system covers the concept of epidemical transmutation (metallaxis in Greek). New information that is locally inferred (through conceptual reasoning) becomes a potential epidemic, which also propagates through the network.

Epidemical algorithms support the spreading of information among nearby nodes. There has been considerable research related to information dissemination in mobile, adhoc networks. The architecture in [5] uses the mobility of users for multimedia files transfer between separate networks thus there is no support for collaborative contextawareness.Additionally, the architecture discussed in [3] proposes an approach for collaborative context dissemination among groups of mobile terminals. Such architecture takes into account the reliability of contextual information in the dissemination process. Nevertheless, the information spreading does not adopt epidemiological models. Instead, a simple flood based model is adopted. Moreover, the autonomous gossiping algorithm in [1] refers to the selectivity attribute of the epidemical spreading. However, such algorithm does not consider any reasoning process, thus, nodes cannot reason with semantic information and, consequently, cannot augment the knowledge in a collaborative context-aware system.

This paper is organized as follows: in Section 2 the characteristics related to semantic information dissemination and certain knowledge representation issues are discussed. In Section 3 an epidemiological model based on Markov processes is introduced, while in Section 4 analytical and simulation results are presented. Finally, in Section 5 conclusions and directions for future work are presented.

\section{SEMANTIC DISSEMINATION}

Semantics-based information dissemination incarnates diffusion aspects for semantic information representation and difussion. Therefore, the questions issued in collaborative context - aware environments are (i) whether semantic information dissemination demonstrates an epidemic-like propagation pattern and (ii) whether such dissemination is affected by: (1) the reasoning capability of the nodes, (2) the network topology (e.g., homogeneous and scale-free topologies), (3) the network infrastructure (e.g., ad - hoc mobile networks), (4) the mobility behavior of nodes (i.e., stationary and mobile nodes) and (5) the knowledge representation (e.g., metadata is represented by hierarchically structured ontological concepts). Therefore, mobile ad-hoc communication is considered more advantageous with respect to infrastructure-based communication approaches, where (i) no global coordination is needed, (ii) cost is reduced (no infrastructure is needed) and (iii) the coverage of the network can be extended through multi-hop communication. However, such lack of infrastructure brings some challenges to the development of collaborative epidemical algorithms. The development of such algorithms requires the cooperation of mobile nodes on a local basis in order to achieve a global goal. Such requirement includes the study of information dissemination schemes and the distributed nature of ad-hoc networks. Moreover, nodes must autonomously take decisions based on information provided by the neighboring environment. The following sections describe the requirements and properties of semantic information dissemination.

\subsection{Dissemination Characteristics}

Infrastructureless Network:The requirement of cooperation of participating nodes brings an additional limitation using multicast in ad-hoc networks. Not all nodes participating in a multicast tree are interested in the diffused information. Cooperation exists only in the sense that, nodes diffuse information to others that are of similar interest. The proposed model has to be suitable for ad-hoc networks because of its self-organizing nature rather than reliance on infrastructure or maintenance of routing information. Such type of dissemination is resilient to sudden failures of links or nodes[2]. Due to its stateless nature, epidemical spreading is not affected by node mobility, as complex, stateful, distributed algorithms are (e.g., routing protocols).

Network Topology Reliance:The performance of epidemical spreading (reliability and efficiency) is strongly affected by the connectivity patterns of the underlying network topology. Nodes are more likely to spread information across small world networks than across networks with many redundant connections. Epidemical dissemination appears to be reliable in homogenous network topology. On the other hand, information spreading in scale-free topology performs more efficiently in terms of the network load.

Semantics-based Dissemination: Semantics-based epidemical dissemination means that, if two nodes, whose hierarchically structured pieces of information are complementary, missing or associated with generalization relations (e.g., $\sqsubseteq$ relations), come in contact with each other, they probably diffuse their knowledge. This does not imply that, nodes always get infected with the information they desire (as succeeded in Flooding). Instead, they are not spammed by multiple and unnecessary data. Such requirement demands dissemination schemes based on knowledge reasoning rather than network-based communication. Moreover, the reasoning about semantically enriched information (i.e., a conceptual ontology is assumed) results in knowledge diffusion. A more abstract concept, e.g., Blues music genre, is less usable than a more specific (detailed) concept, e.g., Rhythm and Blues or Soul music genres, i.e., Soul $\sqsubseteq$ Rhythm and Blues $\sqsubseteq$ Blues [4]. That is attributed to the fact that, the latter concepts convey more detailed information than the former. Evidently, the knowledge derived from the latter concepts implies also the knowledge derived from the former. Semantics-based dissemination is mainly based on locally available knowledge and autonomous reasoning. If a semantic annotation of a multimedia content corresponds to more detailed concept (analogous to a stronger epidemic) then, it tries to (re)infect as many neighboring nodes as possible with similar interests. Consequently, each node attempts to be infected by stronger epidemics.

Selectivity Attribute: A node can autonomously infer if the incoming epidemic refers to a multimedia content that adequately matches to his preference or not. If the incoming information is inconsistent with his interest then, a node avoids processing it. The reliability of the spreading process depends on the suitability of nodes to reason about semantic information.

Double-epidemical Dissemination: The proposed epidemical model is, essentially, an epidemic algorithm but unlike previ- 
ous usage for broadcast, the model is selective in epidemical spreading and deals with numerous pieces of semantic information. Each piece of information is regarded as a different epidemic and transmuted epidemics spread in the network simultaneously. Each epidemic autonomously infects nodes that are susceptible to such kind of epidemic or susceptible to the transmuted one. Hence, a double-epidemical (epidemic and its transmutation) propagation process is considered. In this sense, the strongest epidemic has the potential to infect a large portion of susceptible nodes, contrary to the weakest epidemic, which infects a small portion of the group. The novelty of the proposed model is the fact that epidemics are semantically dependent through semantic relations in conceptual hierarchies and can transmute to stronger ones (introducing the concept of metallaxis). In the long run, portions of the population are infected either with epidemics or with their transmutations. Consequently, diverse types of semantic information infect the whole network, where each type of information corresponds to the heterogeneous need of each node, as required in the collaborative context-aware systems, i.e., not all nodes is interested in the same multimedia content.

\subsection{Semantic Representation}

Semantic information can be represented by hierarchically structured concepts belonging to domain ontologies. Ontology is the conceptualisation of a world describing taxonomies of concepts induced by generalization relations $(\sqsubseteq)$. Therefore, the desired intelligence in a multimedia context-aware applications is expected to be supported by the exploitation of metadata of the multimedia content. Metadata is expressed by ontological concepts based on the use of knowledge representation and reasoning. Such metadata corresponds to the creation information of the MPEG- 7 standard (i.e., the title and the classification of the movie). MPEG7 is regarded as the most complete specification for multimedia content annotation. Semantic annotation leads to a more formal way that enables more advanced reasoning. The annotation vocabulary is borrowed from relevant domain ontologies (e.g., music ontologies). Moreover, the user preferences could be also represented as ontological concepts sharing similar multimedia annotation vocabularies. The reasoning engine of a node deduces whether a multimedia content mateches a user interest or not.

Let $O$ be a domain ontology that corresponds to the hierarchy of concepts formed by $\sqsubseteq$ relations among them. We refer to an epidemic $p$ as a concept $p(c) \in O$ which semantically describes a multimedia content $c$. Let $\Phi(p)$ be the set of all concepts that are more generic than $p$, that is, $\Phi(p)=\{q \mid p \sqsubseteq q \vee p=q\}$, then $O$ refers to the set of concepts that are associated with transitive $\sqsubseteq$, that is, $O=\{p \sqsubseteq q \mid \Phi(p) \cap \Phi(q) \neq \emptyset\}$. Moreover, a user interest is described by a concept of $r \in O$. Hence, the reasoning engine of a node $i$ deduces that a content $c$ described by an epidemic $p \in O, p(c)$, matches to $r$ iff $\exists p \in O,(r \sqsubseteq p)$. If there is a stronger epidemic $q \in O$, i.e., $q \sqsubseteq p$, then the node $i$ is infected with $q$ iff $(q \sqsubseteq r \wedge q \sqsubseteq p)$. We call $q$ as a transmutation $\succ$ of $p$, i.e., $q \succ p$, since node $i$ is reinfected with $q$.

\section{ANALYSIS}

We adopt the epidemiological model Susceptible-InfectedSusceptible (SIS) in which, infectious nodes are those that have contracted the epidemic and can infect the remaining susceptible ones. After a period of time, infected nodes may recover from the epidemic and then transit to the susceptible state. In that state, they can become infected again, thus, in the limit, any node perpetually moves between the two states: Susceptible - Infected. We extend such model at the point that, an infected node can be reinfected with a transmuted epidemic.

\subsection{Notation}

We use a directed graph $\mathcal{G}(V, E)$ to represent a doubleepidemical network, where $V$ is the set of nodes and $E$ is the set of edges. In order to illustrate the concept of epidemical transmutation, we assume that, an epidemic is transmuted only to a stronger epidemic. Let us denote the state of node $i$ at time instant $t$ by $x_{i}(t)$. This state assumes 3 values which are represented by the 3 -dimensional vectors $p_{0}=[100]^{T}$, $p_{1}=[010]^{T}$ and $p_{2}=[001]^{T}$. A state of value $p_{k}$ denotes that the node is in infectious status of level $k$. A node with susceptible status is in a state $p_{0}$ whereas a node with the most infectuous status is in a state $p_{2}$. A neighborhood of node $i$, denoted by $V_{i}$, is a subset of $V$ where every node $j$ in this subset has an edge connecting it to node $i$, i.e., $V_{i}=\{j \mid(j, i) \in E\}$. Each edge $(j, i)$ in $E$ is associated with $\beta_{j i}$, which is the birth rate that an infected node $j$ can infect a neighbor node $i$. For the values of a state of a node we assume an ordering in the form of $p_{2} \succ p_{1} \succ p_{0}$. This means that epidemic $p_{2}$ is stronger than epidemic $p_{1}$ and state $p_{1}$ is stronger than the susceptible state $p_{0}$. A node infected by $p_{1}$ is probable to re-infected by $p_{2}$ as this stronger epidemic spreads across the network. Probabilistic double-epidemical spreading deals with the calculation of the expected number $n_{k}(t)$ of infected nodes for all transmuted epidemics $p_{k}$, $k=1,2$. The concept of epidemic transmutation assumes that an infectious node $i$ of infection level $p_{l}$, can only infect a node $j$ in its neighborhood, which is in state $p_{k}$, if and only if $p_{l} \succ p_{k}$, that is either node $j$ is susceptible $\left(p_{0}\right)$ or it is infected at a lower level.

An infectious node $i$ of infection level $p_{2}$ can be cured in two ways. In the full cure transition case, node $i$ transits in one step to the susceptible state $p_{0}$ with rate $\epsilon$. In the partial cure transition case, node $i$, being in state $p_{2}$, transits in the lower infectious state $p_{1}$ with rate $\delta$. Partially cure means that, the node may be still infectious since it transits to a state corresponding to a weaker epidemic than the previous one. Figure 1 depicts the state transition diagram of a node. It should be noted that the transition rates depend on the number of neighbor infected nodes, their infection level and the birth rate beta $a_{i j}$. This will be further clarified in the next subsection.

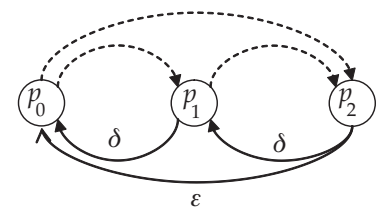

Figure 1: State tranistion diagram of a node. 


\subsection{Spatio-Temporal Model}

As node $i$ can be infected only by its neighbors, the state $x_{i}(t)$ is statistically dependent on the status of its neighbors and $x_{i}(t-1)$. Since the status of a neighbor also depends on its own neighbors, then, the status of all nodes is statistically dependent in space and time. Let vector $\mathbf{x}(t)$ denote the status of all nodes at time $t$, that is

$$
\mathbf{x}(t)=\left[x_{1}(t), x_{2}(t), \ldots, x_{M}(t)\right]^{T}
$$

where $M$ is the number of nodes in the network; $\mathbf{x}(t)$ is a spatiotemporal process. The infection and recovery rates $\beta_{j i}$ and $\delta, \epsilon$ respectively, are very important in the epidemiological epistemic domain. The infection rate $\beta_{j i}$ denotes the birth rate of an epidemic from node $j \in V_{i}$. Given the status of the neighbors of node $i$ at time instant $t$ and the fact that node $i$ may be infectious at level $k$, at the next time instant $t+1$ node $i$ will be infectious at a higher level $l$ with probability

$$
\begin{aligned}
Q_{k l}= & \left(1-\sum_{m<k} \delta_{k m}\right) \cdot\left(1-\prod_{j \in V_{i}}\left(1-\beta_{j i}\right)^{x_{j}^{T}(t) \cdot p_{l}}\right) \\
& \prod_{j \in V_{i}}\left(1-\beta_{j i}\right)^{x_{j}^{T}(t) \cdot \sum_{m>l} p_{m}}
\end{aligned}
$$

The second line in (1) expresses the probability that all the nodes $j \in V_{i}$ with an infection level greater than $l$ will not infect node $i$. The expression in the parenthesis in the first line in (1) is the probability that one or more nodes will infect node $i$ at infection level $l$ and node $i$ will not recover. In view of the state transition diagram of Figure 1, considering a Markov chain of unit time transition periods, the transition probabilities that express the temporal dependence of states of node $i$ are Full cure case:

$$
\begin{aligned}
& P\left\{x_{i}(t+1)=p_{0} \mid x_{i}(t)=p_{1}\right\}=\delta \\
& P\left\{x_{i}(t+1)=p_{0} \mid x_{i}(t)=p_{2}\right\}=\epsilon
\end{aligned}
$$

Partial cure case:

$$
P\left\{x_{i}(t+1)=p_{1} \mid x_{i}(t)=p_{2}\right\}=\delta
$$

Infection at a higher level $k<l$

$$
P\left\{x_{i}(t+1)=p_{l} \mid X_{V_{i}}(t)=\mathbf{x}_{V_{i}}(t), x_{i}(t)=p_{k}\right\}=Q_{k l}
$$

where, the random vector $X_{V_{i}}(t)$ denotes the status of all neighbors of node $i$, i.e., $X_{V_{i}}(t)=\left[x_{j}(t), j \in V_{i}\right]$ and $\mathbf{x}_{V_{i}}(t)$ is a realization of $X_{V_{i}}(t)$. Given the conditional probabilities in Equations (2)-(5) we can calculate the probability that a node $i$ is in state $p_{k}$ at time instant $t+1$, that is

$$
\begin{aligned}
& P\left\{x_{i}(t+1)=p_{k}\right\} \\
&= \sum_{p_{m}} P\left\{x_{i}(t+1)=p_{k}, x_{i}(t)=p_{m}\right\} \\
&= \sum_{p_{m}} P\left\{x_{i}(t+1)=p_{k} \mid x_{i}(t)=p_{m}\right\} P\left\{x_{i}(t)=p_{m}\right\} \\
&= \sum_{p_{m}} \sum_{\mathbf{x}_{V_{i}}(t)} P\left\{x_{i}(t+1)=p_{k}, X_{V_{i}}(t)=\mathbf{x}_{V_{i}}(t) \mid x_{i}(t)=p_{m}\right\} \\
& \cdot P\left\{x_{i}(t)=p_{m}\right\}
\end{aligned}
$$

and, finally,

$$
\begin{aligned}
& P\left\{x_{i}(t+1)=p_{k}\right\}= \\
& \quad \sum_{p_{m}} \sum_{\mathbf{x}_{V_{i}}(t)} P\left\{x_{i}(t+1)=p_{k} \mid X_{V_{i}}(t)=\mathbf{x}_{V_{i}}(t), x_{i}(t)=p_{m}\right\} \\
& \cdot P\left\{X_{V_{i}}(t)=\mathbf{x}_{V_{i}}(t) \mid x_{i}(t)=p_{m}\right\} P\left\{x_{i}(t)=p_{m}\right\}
\end{aligned}
$$

The conditional probability

$$
P_{m}(t)=P\left\{X_{V_{i}}(t)=\mathbf{x}_{V_{i}}(t) \mid x_{i}(t)=p_{m}\right\}
$$

characterizes explicitly the spatial statistical dependencies due to network topology and nodal interactions. To simplify the analysis we adopt a spatial independence assumption. For spatially independent nodes

$$
\begin{aligned}
P_{m}(t) & =P\left\{X_{V_{i}}(t)=\mathbf{x}_{V_{i}}(t) \mid x_{i}(t)=p_{m}\right\} \\
& =P\left\{X_{V_{i}}(t)=\mathbf{x}_{V_{i}}(t)\right\}=\prod_{j \in V_{i}} P\left\{x_{j}(t)\right\}
\end{aligned}
$$

Although the independence assumption ignores the spatial dependence of nodes, the model maintains temporal dependency and detailed topology information. Moreover, if node $i$ has $\left|V_{i}\right|$ neighbors, the total number of states needed to describe $P_{m}(t)$ is reduced form $O\left(3^{\left|V_{i}\right|}\right)$ to $O\left(\left|V_{i}\right|\right)$. We now focus on the calculation of the probabilities $P\left\{x_{i}(t+1)=p_{0}\right\}$, $P\left\{x_{i}(t+1)=p_{1}\right\}$ and $P\left\{x_{i}(t+1)=p_{2}\right\}$. To simplify the analysis we assume that $\beta_{j i}=\beta$. Based on Equations (6) and (8) we have

$$
\begin{aligned}
& P\left\{x_{i}(t+1)=p_{0}\right\}= \\
& \sum_{\mathbf{x}_{V_{i}}(t)} Q_{00} \prod_{j \in V_{i}} P\left\{x_{j}(t)\right\} \cdot P\left\{x_{i}(t)=p_{0}\right\} \\
& \quad+\delta \cdot P\left\{x_{i}(t)=p_{1}\right\}+\varepsilon \cdot P\left\{x_{i}(t)=p_{2}\right\}
\end{aligned}
$$

where

$$
Q_{00}=\prod_{j \in V_{i}}(1-\beta)^{x_{j}^{T}(t)\left(p_{1}+p_{2}\right)}
$$

Using the fact

$$
\sum_{\mathbf{x}_{V_{i}}(t)} \prod_{j \in V_{i}} f\left(x_{j}(t)\right)=\prod_{j \in V_{i}} \sum_{x_{j}(t)} f\left(x_{j}(t)\right)
$$

Equation (9) simplifies to

$$
\begin{aligned}
& P\left\{x_{i}(t+1)=p_{0}\right\} \\
& =\prod_{j \in V_{i}}\left(1-\beta+\beta P\left\{x_{j}(t)=p_{0}\right\}\right) \cdot P\left\{x_{i}(t)=p_{0}\right\} \\
& \quad+\delta \cdot P\left\{x_{i}(t)=p_{1}\right\}+\varepsilon \cdot P\left\{x_{i}(t)=p_{2}\right\}
\end{aligned}
$$

For the probability $P\left\{x_{i}(t+1)=p_{1}\right\}$ we have

$$
\begin{aligned}
& P\left\{x_{i}(t+1)=p_{1}\right\}= \\
& \sum_{\mathbf{x}_{V_{i}}(t)} Q_{01} \prod_{j \in V_{i}} P\left\{x_{j}(t)\right\} \cdot P\left\{x_{i}(t)=p_{0}\right\}+ \\
& \sum_{\mathbf{x}_{V_{i}}(t)} Q_{11} \prod_{j \in V_{i}} P\left\{x_{j}(t)\right\} \cdot P\left\{x_{i}(t)=p_{1}\right\}+\delta \cdot P\left\{x_{i}(t)=p_{2}\right\}
\end{aligned}
$$

where $Q_{01}$ is given by (1) and

$$
Q_{11}=(1-\delta) \prod_{j \in V_{i}}(1-\beta)^{x_{j}^{T}(t) \cdot p 2}
$$


Using the same arguments as for the calculation of $P\left\{x_{i}(t+\right.$ 1) $\left.=p_{0}\right\}$, we obtain

$$
\begin{aligned}
& P\left\{x_{i}(t+1)=p_{1}\right\}= \\
& \quad \prod_{j \in V_{i}}\left(1-\beta P\left\{x_{j}(t)=p_{2}\right\} P\left\{x_{i}(t)=p_{0}\right\}\right. \\
& \quad-\prod_{j \in V_{i}}\left(1-\beta+\beta P\left\{x_{j}(t)=p_{0}\right\} P\left\{x_{i}(t)=p_{0}\right\}\right. \\
& \quad+(1-\delta) \prod_{j \in V_{i}}\left(1-\beta P\left\{x_{j}(t)=p_{2}\right\}\right) \cdot P\left\{x_{i}(t)=p_{1}\right\} \\
& \quad+\delta \cdot P\left\{x_{i}(t)=p_{2}\right\}
\end{aligned}
$$

Finally,

$$
\begin{aligned}
& P\left\{x_{i}(t+1)=p_{2}\right\}= \\
& \sum_{\mathbf{x}_{V_{i}}(t)} Q_{02} \prod_{j \in V_{i}} P\left\{x_{j}(t)\right\} \cdot P\left\{x_{i}(t)=p_{0}\right\}+ \\
& \sum_{\mathbf{x}_{V_{i}}(t)} Q_{12} \prod_{j \in V_{i}} P\left\{x_{j}(t)\right\} \cdot P\left\{x_{i}(t)=p_{1}\right\}+ \\
& \quad(1-\delta-\epsilon) \cdot P\left\{x_{i}(t)=p_{2}\right\}
\end{aligned}
$$

from which we obtain

$$
\begin{aligned}
& P\left\{x_{i}(t+1)=p_{2}\right\}= \\
& \quad\left(1-\prod_{j i n V_{i}}\left(1-\beta P\left\{x_{j}(t)=p_{2}\right\}\right) P\left\{x_{i}(t)=p_{0}\right\}\right. \\
& \quad+(1-\delta) \cdot\left(1-\prod_{j i n V_{i}}\left(1-\beta P\left\{x_{j}(t)=p_{2}\right\}\right) P\left\{x_{i}(t)=p_{1}\right\}\right. \\
& \quad+(1-\delta-\epsilon) \cdot P\left\{x_{i}(t)=p_{2}\right\}
\end{aligned}
$$

\section{ANALYTICAL \& SIMULATION RESULTS}

We assess the behavior of the double-epidemical dissemination model in homogeneous and scale-free networks. As a reference of a homogeneous network, we consider a regular two-dimensional (2D) lattice. Nodes in a 2D lattice are only connected with neighbors. A node in a $2 \mathrm{D}$ lattice is represented by its cordinates $(x, y)$, where $x, y$, are integers and $1 \leq x, y \leq 100$. Node $(x, y)$ has four neighbors $(x-1, y)$, $(x+1, y),(x, y-1),(x, y+1)$ thus, the average node degree $\langle k\rangle$ for a $2 \mathrm{D}$-lattice is $\langle k\rangle=4$. The nodes at the borders have node degree less than $\langle k\rangle$. Figure 2 shows the evolution of the average number of infected nodes $n_{1}(t), n_{2}(t)$ for two epidemics $p_{1}$ and $p_{2}, p_{2} \succ p_{1}$, for the analytical epidemiological model with $M=10,000$ nodes, $\beta=0.2$, $\delta=0.1$ and $\epsilon=0.01$. The semantic relationship among epidemics demonstrates an interesting behavior. Evidently, the strongest epidemic infects the largest portion of the network since more specific information, which is inferred by nodes, is closer to users' interests. At the beginning of the propagation process $n_{2}(t)$, which refers to the most specific concept, assumes lower value than $n_{1}(t)$, which refers to the most generic one. In the long run, more knowledge is accumulated across the collaborating nodes thus $n_{2}(t)$ dominates $n_{1}(t)$. This is attributed to the fact that, since nodes reason about more specific knowledge then, they are reinfected with the strongest epidemic assuming that the latter matches better to their interests. The higher the value of

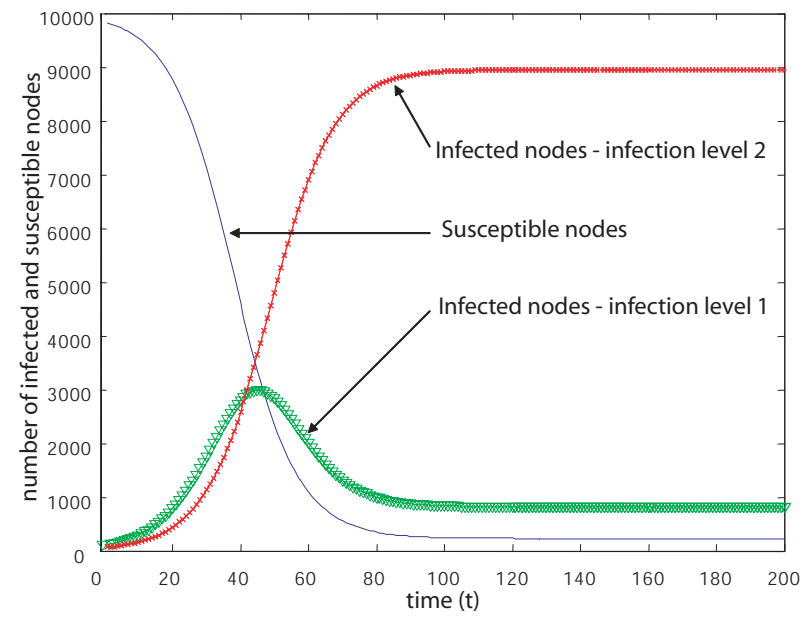

Figure 2: Double-epidemical propagation in 2D lattice with $\beta=0.2, \delta=0.1$ and $\epsilon=0.01$

$\beta$, w.r.t. $\delta$ and $\epsilon$, i.e., there are more nodes with reasoning capability, the faster the network gets infected.

Figure 3 depicts the evolution of the propagation process for a $2 \mathrm{D}$ lattice with $M=10,000$ nodes for $\beta=0.2, \delta=0.1$ and $\epsilon=0.6$. One could observe that, if the full cure rate of the strongest epidemic, $\epsilon$, is relatively larger than $\delta$, (e.g., a minor portion of nodes are capable of reasoning) then, the propagation process for $p_{2}$ decays. This does not necessarily hold true for the propagation process of $p_{1}$. Instead, $p_{1}$ cannot trasmute to a stronger epidemic due to the limited reasoning capability of the majority of nodes. However, the existence of $p_{2}$ in a network depends highly on the fact that (i) at least one node is capable of inferring $p_{2}$ from $p_{1}$ or (ii) at least a node is infected with $p_{2}$ at the beginning of the process. We also examine the behavior of double-epidemical

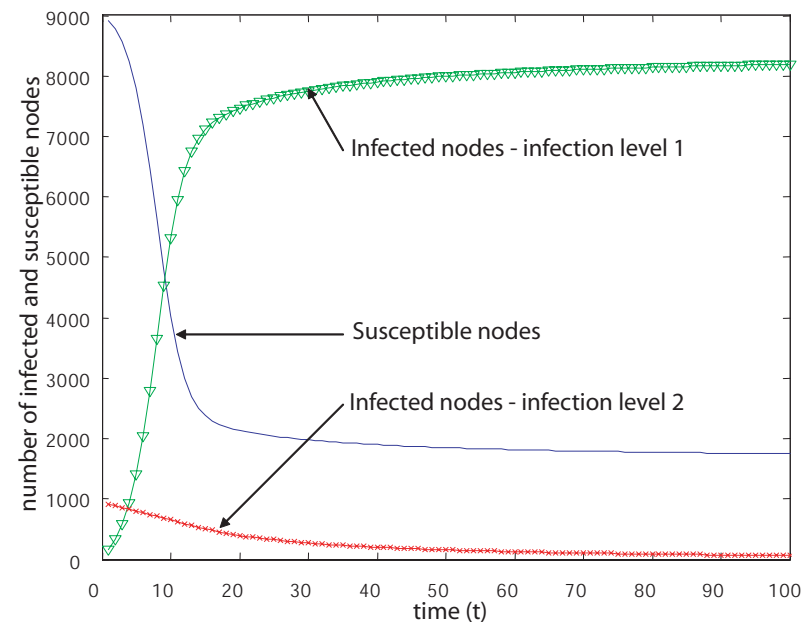

Figure 3: Double-epidemical propagation in 2D lattice with $\beta=0.2, \delta=0.1$ and $\epsilon=0.6$

spreading in scale-free networks. In such networks the prob- 
ability for a node $i$ to be connected to a neighboring node $j$ of degree $k_{j}$ depends on its own degree $k_{i}$. We use a scale - free network topology of the Oregon ${ }^{1}$ routers network type. Such network type contains 22002 connections among 9895 peers with $\langle k\rangle=92.61$. Figure 4 depicts an analogous behavior of the proposed model with that in homogeneous networks. Epidemics propagation in complex networks appears highly correlated to the existence of highly connected nodes. Therefore, diverse epidemical thresholds can appear in such networks based on the value of $\beta, \delta$ and $\epsilon$, but the study of this issue is beyond the scope of this paper. Figure

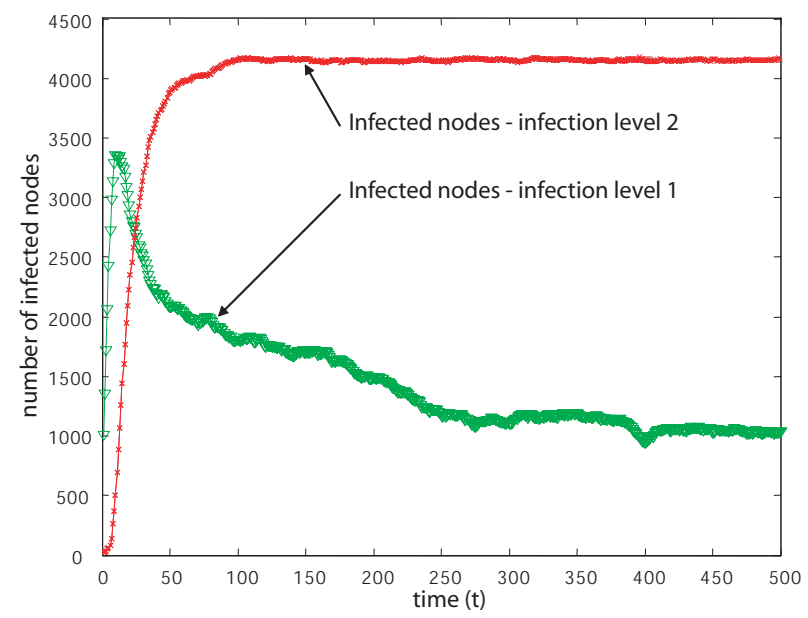

Figure 4: Double-epidemical propagation in scalefree network with $\beta=0.095, \delta=0.03$ and $\epsilon=0.02$

5 shows the simulation results of the evolution of the proposed model on a four-neighbor two-dimensional lattice with 10,000 nodes, $\beta=0.03, \delta=0.01$ and $\epsilon=0.002$. The discrepancies between the simulaton results and the analytical model are attributed to the fact that, nodes at the borders of the 2D lattice have a low value of node degree. However, the proposed model based on the spatial independency of nodes describes adequately the transient behavior of doubleepidemical propagation.

\section{CONCLUSIONS}

We propose an epidemiological model for multimedia semantics information dissemination. We use ontological representation for both modeling multimedia content metadata and user interests. A user receives the desired multimedia content that matches his interests once the corresponding semantic description (epidemic) propagates across the network. Our model goes beyond a simple epidemiological model and introduces the abstraction of a stronger and transmuted epidemic. We extend the SIS model defining the concept of epidemical transmutation in information dissemination. A probabilistic model is introduced describing a double-epidemical dissemination model and analytical and simulation results are reported. In most models, semantic information dissemination is not considered thus, the reasoning capability is not efficient. The proposed

\footnotetext{
$\overline{{ }^{1} \text { http://topology.eecs.umich.edu/data/html }}$
}

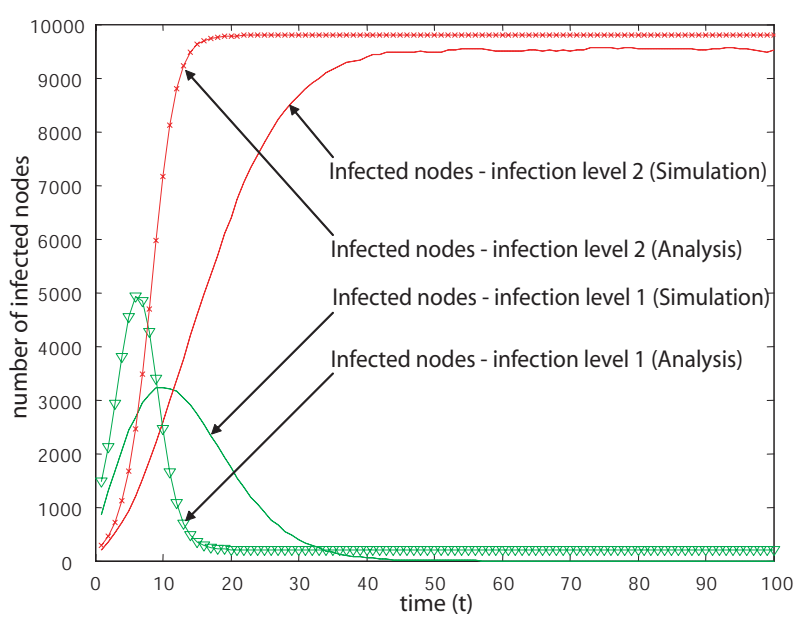

Figure 5: Simulation of double-epidemical propagation in 2D lattice network with $\beta=0.03, \delta=0.01$ and $\epsilon=0.002$

model is novel because, the epidemic, which might be transmuted, can reinfect the nodes thus aggravating their condition. However, issues related to the epidemical thresholds and the network topologies have to be examined. Moreover, a multiple-epidemical information dissemination model, in which an epidemic transmutes to more than one stronger epidemic, are another interesting area we are currently working on.

\section{REFERENCES}

[1] A. Datta, S. Quarteroni, and K. Aberer. Autonomous gossiping: A self-organizing epidemic algorithm for selective information dissemination in wireless mobile ad-hoc networks. In Semantics of a Networked World, pages 126-143, 2004.

[2] D. Kempe, J. Kleinberg, and A. Demers. Spatial gossip and resource location protocols. Journal of the ACM, 51(6):943-967, November 2004.

[3] J. Mantyjarvi, P. Huuskonen, and J. Himberg. Collaborative context determination to support mobile terminal applications. In Pervasive Communications, pages 161-168, 2003.

[4] F. Pachet and D. Cazaly. A taxonomy of musical genres. In Content-Based Multimedia Information Access (RIAO) Conference, 2000.

[5] P. Tennert, M. Hall, B. Brown, M. Chalmers, and S. Sherwood. Three applications for mobile epidemic algorithms. In Human Computer Interaction with Mobile Devices and Services, pages 223-226, 2005. 